\title{
Access to healthcare during COVID-19 among ageing population in Latvia
}

\author{
Ieva Reine $^{1, *}$, Madara Mikelsone ${ }^{1}$, Andrejs Ivanovs ${ }^{1}$, and Signe Tomsone ${ }^{2}$ \\ ${ }^{1}$ Statistics Unit, Riga Stradins University, Riga, Latvia \\ ${ }^{2}$ Department of Rehabilitation, Riga Stradins University, Riga, Latvia
}

\begin{abstract}
Medical staff has worked tirelessly throughout the COVID-19 pandemic to test and treat coronavirus patients. However, the focus on the virus has caused huge disruption to healthcare services, resulting in delayed treatment for patients with other serious conditions. Additionally, the reasons for forgoing or abstaining healthcare could also relate to individual decisions. We used data from the Survey of Health, Ageing and Retirement in Europe (SHARE), where the respondents aged 50 and older of the Wave 8 , version 0 , were asked to answer, among other, questions on delayed, postponed or denied healthcare services during the first wave of COVID-19 pandemic in Latvia. For comparative reasons also Estonian and Lithuanian data were included in the study with 2473627 valid weighted cases in total. Due to the large sample size, in order to evaluate statistically significant result for Pearson's chi-square test, strength of association (f) was provided. The results show that $12.7 \%$ of the respondents forwent and $14 \%$ postponed medical treatment in Latvia. Appointment was denied to $5.5 \%$ of the respondents. Generally, older people in Latvia had better access to health care services compared to the average in the Baltic countries. We found significant age and gender differences, but they varied depending on the type of treatment and reason for not receiving it. However, further studies are necessary to analyse how limited access to the healthcare for other reasons has affected health of the ageing population. Also, more knowledge is needed about how to maintain public health and wellbeing for the growing part of the ageing society.
\end{abstract}

\section{Introduction}

Since the outbreak of COVID-19, the focus has mainly been on morbidity and mortality of the most vulnerable populations, and the elderly received special attention as one of the groups exposed to the highest health risks associated with the pandemic [1]. Recent research on COVID-19 effects stresses that inequities and discriminations are also important risk factors for overall poor health and decreased life expectancy [2]. Furthermore, other studies have also shown that there is a relationship between a degree of inequity and extent of social segregation. It could lead to longer time to detect outbreaks and tackle the consequences of COVID-19 among specific age groups, like the elderly [3,4]. The most affected part of the population with co-morbidities has been found as powerful predictors of hospitalization;

\footnotetext{
${ }^{*}$ Corresponding author: ieva.reine@ rsu.lv
} 
however, admission oxygen impairment and markers of inflammation are most strongly associated with critical illness [5].

Medical staff has worked tirelessly throughout the COVID-19 pandemic to test and treat coronavirus patients. However, restrictions necessary to prevent the spread of the virus affected the use of healthcare services for conditions unrelated to COVID-19 [6,7]. Thus, the lack of attention to multi-morbidity in older people, due to the focus on COVID-19, might have both short as well as long-term health effects for those who forwent, postponed or were denied different healthcare services.

People at risk to develop serious conditions from COVID-19 are to large extent those with cardiovascular diseases, overweight, with hypertension and diabetes [6]. In Latvia, more than a half of the population aged 50+ has at least one of these conditions. Among those aged 65 and above, almost one in five has more than two chronic conditions requiring timely treatment. Thus, public health research is needed to understand and mitigate both the impact of COVID-19 and beyond it for those with underlying conditions. Highlighting the COVID19 pandemic effects on ageing populations would be useful to address systemic and major challenges such as socioeconomic and health inequalities, and sustainable futures [8].

This study raises awareness that the important focus on those suffering and dying from COVID-19 should be expanded to address wider questions on the inequalities in access to healthcare during COVID-19 among older people. The aim of the study was to analyse if there were interruptions or problems to access healthcare during the first wave of COVID-19 among ageing population in Latvia.

\section{Material and method}

The analysis is based mainly on data of Latvia, but for comparative reasons also on data of Estonia and Lithuania derived from Wave 8, version $0[9,10]$ of the international longitudinal Survey of Health, Ageing and Retirement in Europe (SHARE) ${ }^{1}$. The study acquires comparable data on people aged 50 and older: their daily lives, health and social life circumstances, views, values and habits. Two surveys were administered within the scope of Wave 8: data was obtained before the COVID-19 outbreak and after the first wave of the outbreak in the summer of 2020, reaching 1207 respondents in Latvia. Respondents of the age 50+ were asked to answer questions on forgone, delayed or denied healthcare services during the first wave of Covid-19 pandemic in Latvia. The study is based on 2473627 valid weighted cases in the Baltic countries. Pearson's chi-squared test was performed to assess the differences between the age groups, men and women. Due to a large sample size, to evaluate statistically significant result for Pearson's chi-square test, strength of association (f) was provided. Analyses were carried out using the statistical package SPSS (Version 27) on preliminary SHARE Wave 8 release 0 data, thus, the results are preliminary and should be interpreted with caution.

\section{Results}

The outbreak of COVID-19 caused limited access to healthcare in many countries. Figure 1 shows the proportion of those aged 50+ who forwent, postponed or were denied medical appointment in Latvia, and the mean percentage in the Baltic countries - Latvia, Estonia and Lithuania. It was more common to postpone medical appointment (14\%), which is considerably less than the average in the Baltic countries (22.7\%). Results indicate that $12.7 \%$

\footnotetext{
${ }^{1}$ This paper uses data from Wave 8 (DOI: 10.6103/SHARE.w8cabeta.001), see Börsch-Supan et al. (2013) for methodological details. The SHARE data collection has been funded by the European Commission.
} 
forwent medical treatment, and it does not considerably differ from the average. A smaller proportion of the respondents in Latvia $-5.5 \%$, were denied medical appointment compared to $8.8 \%$ in the Baltic countries.

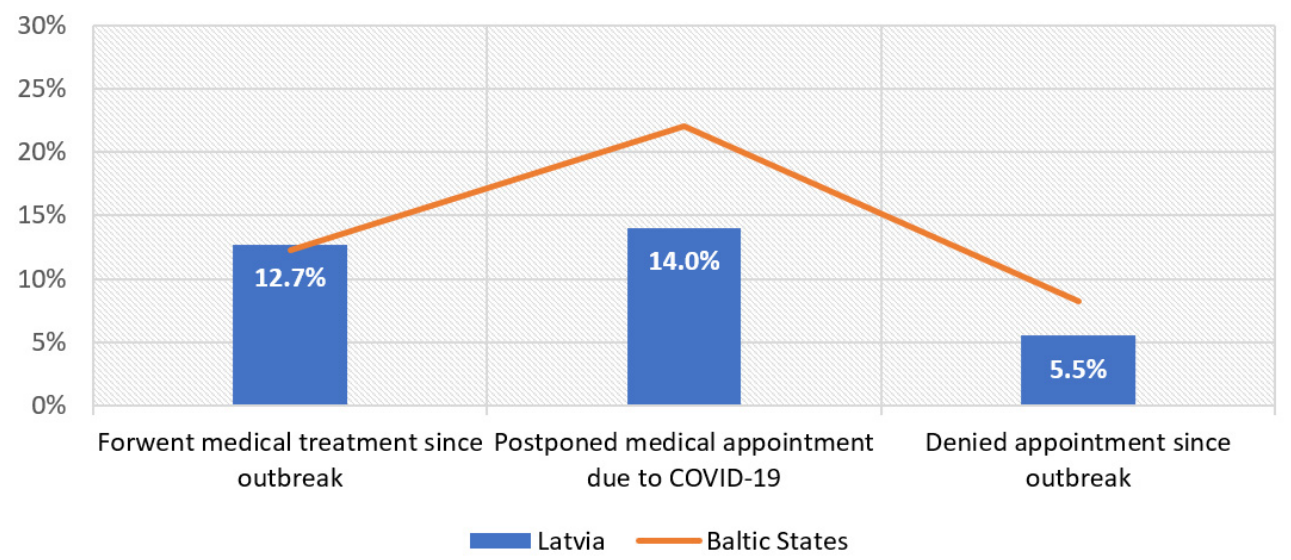

Figure 1. Comparison of forwent, postponed and denied medical treatment between Latvia and the Baltic countries.

Table 1 shows the distribution of those in the age groups 50-64 and 65+ who forwent, postponed or were denied medical treatment in Latvia. Significant age differences as regards healthcare service consumption were found for almost all factors. However, no systematic inequalities were found among the age groups.

Table 1. Comparison between age groups and forwent, postponed and denied medical treatment.

\begin{tabular}{|c|c|c|c|c|}
\hline & \multirow{2}{*}{ Factor } & & Age groups & \multirow{2}{*}{$\mathrm{P}$-value $/ \varphi$} \\
\hline & & & $50-64 \quad 65+$ & \\
\hline \multirow{6}{*}{ Forwent } & medical treatment since outbreak & Yes/No & $10.5 \% / 89.5 \% \quad 14.6 \% / 85.4 \%$ & $<0.001 /-0.062$ \\
\hline & general practitioner check up & Yes/No & $55.9 \% / 44.1 \% \quad 83.0 \% / 17.0 \%$ & $<0.001 /-0.296$ \\
\hline & specialist/dentist check up & Yes/No & $90.8 \% / 9.2 \% \quad 64.7 \% / 35.3 \%$ & $<0.001 / 0.294$ \\
\hline & planned medical treatment or operation & Yes/No & $27.0 \% / 73.0 \% \quad 11.7 \% / 88.3 \%$ & $<0.001 / 0.195$ \\
\hline & physiotherapy, psychotherapy, rehabilitation & Yes/No & $20.8 \% / 79.2 \% \quad 4.6 \% / 95.4 \%$ & $<0.001 / 0.253$ \\
\hline & other medical treatment & Yes/No & $38.4 \% / 61.6 \% \quad 14.6 \% / 85.4 \%$ & $<0.001 / 0.272$ \\
\hline \multirow{6}{*}{ Postponed } & medical appointment due to COVID-19 & Yes/No & $14.3 \% / 85.7 \% \quad 13.7 \% / 86.3 \%$ & $<0.001 / 0.009$ \\
\hline & general practitioner check up & Yes/No & $23.7 \% / 76.3 \% \quad 27.9 \% / 72.1 \%$ & $<0.001 /-0.048$ \\
\hline & specialist/dentist check up & Yes/No & $67.8 \% / 32.2 \% \quad 72.2 \% / 27.8 \%$ & $<0.001 /-0.048$ \\
\hline & planned medical treatment or operation & Yes/No & $13.2 \% / 86.8 \% \quad 15.8 \% / 84.2 \%$ & $<0.001 /-0.037$ \\
\hline & physiotherapy, psychotherapy, rehabilitation & Yes/No & $7.3 \% / 92.7 \%$ & $<0.001 / 0.032$ \\
\hline & other medical treatment & Yes/No & $35.3 \% / 64.7 \% \quad 26.4 \% / 73.6 \%$ & $<0.001 / 0.096$ \\
\hline \multirow{6}{*}{ Denied } & appointment since outbreak & Yes/No & $4.6 \% / 95.4 \%$ & $<0.001 / 0.039$ \\
\hline & general practitioner check up & Yes/No & $12.2 \% / 87.8 \% \quad 34.5 \% / 65.5 \%$ & $<0.001 /-0.268$ \\
\hline & specialist/dentist check up & Yes/No & $60.3 \% / 39.7 \% \quad 73.1 \% / 26.9 \%$ & $<0.001 /-0.134$ \\
\hline & planned medical treatment or operation & Yes/No & $12.1 \% / 87.9 \% \quad 12.5 \% / 87.5 \%$ & 0.14 \\
\hline & physiotherapy, psychotherapy, rehabilitation & Yes/No & $8.0 \% / 92.0 \% \quad 13.1 \% / 86.9 \%$ & $<0.001 /-0.083$ \\
\hline & other medical treatment & Yes/No & $21.6 \% / 78.4 \% \quad 18.7 \% / 81.3 \%$ & $<0.001 / 0.036$ \\
\hline
\end{tabular}


The results presented in Table 2 show gender differences, indicating consistent inequalities between men and women regarding different types of healthcare services. Men forwent medical appointments more often than women did, but women were denied check-ups to larger extent than men were.

Table 2. Comparison between gender and forwent, postponed and denied medical treatment.

\begin{tabular}{|c|c|c|c|c|c|}
\hline & \multirow{2}{*}{ Factor } & & \multicolumn{2}{|c|}{ Gender } & \multirow{2}{*}{$\mathrm{P}$-value $/ \varphi$} \\
\hline & & & Men & Women & \\
\hline \multirow{6}{*}{ Forwent } & medical treatment since outbreak & Yes/No & $7.6 \% / 92.4 \%$ & $16.0 \% / 84.0 \%$ & $<0.001 /-0.124$ \\
\hline & general practitioner check up & Yes/No & $61.9 \% / 38.1 \%$ & $75.5 \% / 24.5 \%$ & $<0.001 /-0.129$ \\
\hline & specialist/dentist check up & Yes/No & $77.8 \% / 22.2 \%$ & $74.1 \% / 25.9 \%$ & $<0.001 / 0.036$ \\
\hline & planned medical treatment or operation & Yes/No & $24.5 \% / 75.5 \%$ & $15.7 \% / 84.3 \%$ & $<0.001 / 0.098$ \\
\hline & physiotherapy, psychotherapy, rehabilitation & Yes/No & $18.8 \% / 81.2 \%$ & $8.6 \% / 91.4 \%$ & $<0.001 / 0.137$ \\
\hline & other medical treatment & Yes/No & $23.5 \% / 76.5 \%$ & $24.3 \% / 75.7 \%$ & $0.008 /-0.008$ \\
\hline \multirow{6}{*}{ Postponed } & medical appointment due to COVID-19 & Yes/No & $11.8 \% / 88.2 \%$ & $15.4 \% / 84.6 \%$ & $<0.001 /-0.051$ \\
\hline & general practitioner check up & Yes/No & $24.0 \% / 76.0 \%$ & $26.8 \% / 73.2 \%$ & $<0.001 /-0.030$ \\
\hline & specialist/dentist check up & Yes/No & $73.6 \% / 26.4 \%$ & $68.3 \% / 31.7 \%$ & $<0.001 / 0.055$ \\
\hline & planned medical treatment or operation & Yes/No & $19.6 \% / 80.4 \%$ & $12.0 \% / 88.0 \%$ & $<0.001 / 0.102$ \\
\hline & physiotherapy, psychotherapy, rehabilitation & Yes/No & $16.0 \% / 84.0 \%$ & $4.3 \% / 95.7 \%$ & $<0.001 / 0.200$ \\
\hline & other medical treatment & Yes/No & $26.9 \% / 73.1 \%$ & $32.7 \% / 67.3 \%$ & $<0.001 /-0.059$ \\
\hline \multirow{6}{*}{ Denied } & appointment since outbreak & Yes/No & $6.1 \% / 93.9 \%$ & $5.0 \% / 95.0 \%$ & $<0.001 / 0.023$ \\
\hline & general practitioner check up & Yes/No & $15.1 \% / 84.9 \%$ & $27.6 \% / 72.4 \%$ & $<0.001 /-0.149$ \\
\hline & specialist/dentist check up & Yes/No & $58.9 \% / 41.1 \%$ & $71.6 \% / 28.4 \%$ & $<0.001 /-0.132$ \\
\hline & planned medical treatment or operation & Yes/No & $20.2 \% / 79.8 \%$ & $6.1 \% / 93.9 \%$ & $<0.001 / 0.213$ \\
\hline & physiotherapy, psychotherapy, rehabilitation & Yes/No & $18.0 \% / 82.0 \%$ & $4.2 \% / 95.8 \%$ & $<0.001 / 0.224$ \\
\hline & other medical treatment & Yes/No & $16.0 \% / 84.0 \%$ & $23.7 \% / 76.3 \%$ & $<0.001 /-0.095$ \\
\hline
\end{tabular}

\section{Discussion}

The study shows interruptions and problems in access to healthcare during the first wave of COVID-19 among ageing population in Latvia. A considerable proportion of the population of age 50+ and above in Latvia postponed or forwent medical appointments, thus confirming the results of recent studies showing that the use of healthcare services for conditions unrelated to COVID-19 was seriously affected [9-11].

Still, there were rather few older people in Latvia who were forced to abstain planned medical treatment or operations, as well as physiotherapy, psychotherapy and rehabilitation. However, this study does not have a possibility to answer how a limited access to the healthcare for other reasons has affected health of ageing population and thus remains to be studied. More knowledge is needed about how to maintain public health and wellbeing for this growing part of the society.

The results also indicated that more than one of ten older persons forwent medical treatment, and it could mainly be attributable to persons' own decisions. At the same time, it is notable, that postponed or denied treatments less relatable to personal choices, but rather dependent on the healthcare system, were more beneficial for the older population in Latvia, compared to the average in the Baltic countries. Accordingly, this indicates that the healthcare system in Latvia has been more resilient during the unexpected crisis due to the COVID-19 pandemic in the context of the medical services offered in general in the Baltic countries.

Nevertheless, high costs to receive treatment/consultations at private health care actors might have diminished possibilities to receive medical treatment and offering few alternatives 
to the elderly. The situation also exacerbated the issue of the participation of older people in society remotely. Many of them under-utilize digital solutions in healthcare access and contacts, creating risks of social isolation and ill health. The use of technology varies for older people. In Latvia, digital technologies are used by $65 \%$ of all respondents aged $50+$, but only $36 \%$ use them for healthcare services [9-11], which might have had impact on the possibilities and decisions to receive medical treatment and regular check-ups. More women than men have affordability problems, and thus confirm the health inequalities related to gender. Research also shows that digital skills need to be promoted in an increasingly digital society. The use of technology can significantly increase the independence of older adults and support their daily lives to make health and other services more accessible.

The reasons for forgoing or abstaining healthcare could relate, e.g., to individual decisions, not only to the restrictions or closure of a healthcare institution. While the healthcare system experienced a great challenge, individual decisions and health literacy of the population, [12] have also had an impact on the public health. In line with other studies [7], the obtained results of this study give an opportunity to promote public health and reduce existing inequalities by providing additional motivation for health and health-related behaviour being in line. Our study complies with other recent research [2] that equity-based and personcentred approaches are critical to counter different negative outcomes for vulnerable older population.

The study was performed within the project/agreement No. 1.1.1.2/VIAA/3/19/540 'Challenges of ageing in the Baltic Sea region'.

\section{References}

[1] G. Ksinan Jiskrova, M. Bobák, H. Pikhart, et al. J Epidemiol Community Health Epub ahead of print, DOI:10.1136/jech-2021- 216715 (2021)

[2] L. Dassieu, N. Sourial. Tailoring interventions for social isolation among older persons during the COVID-19 pandemic: challenges and pathways to health care equity. Int $\mathbf{J}$ Equity Health 20, 26 https://doi.org/10.1186/s12939-020-01360-8 (2021)

[3] J.P. Mackenbach, J.R. Valverde, M. Bopp, H. Brфnnum-Hansen, P. Deboosere, R. Kalediene, et al. Determinants of inequalities in life expectancy: an international comparative study of eight risk factors. Lancet Public Health 4(10):e 529-37 (2019)

[4] A. James, M.J. Plank, R.N. Binny, A. Lustig, K. Hannah, S.C. Hendy, N. Steyn, A structured model for COVID-19 spread: modelling age and health care inequities, Mathematical Medicine and Biology: A Journal of the IMA (2021)

[5] C. Petrilli, S. Jones, J.Yang, et al., Factors associated with hospitalization and critical illness among 4,103 patients with COVID-19 disease in New York City. medRxiv (2020)

[6] S. Masroor. Collateral damage of COVID-19 pandemic: delayed medical care. J Card Surg 35, 1345-7 (2020)

[7] O. Oyebode, S.E. Ramsay, C. Brayne. Public health research in the UK to understand and mitigate the impact of COVID-19 and COVID-19 response measures. J Epidemiol Community Healt 75, $209-12$ (2021)

[8] J. Bibby, G. Everest, I. Abbs. The Health Foundation: will COVID-19 be a watershed moment for health inequalities. Available https://www.health.org. uk/publications/longreads/will-COVID-19-be-a-watershed-moment-for-health inequalities (accessed 23 June 2020)

[9] SHARE COVID-19 Release Guide. (2020). http://www.share-project.org/fileadmin/

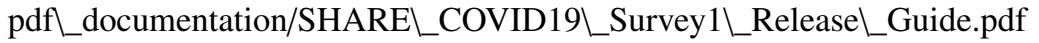


[10] Survey of Health, Ageing and Retirement in Europe, http://www.share-project.org/ (15.05.2021.)

[11] I. Reine, A. Ivanovs, S. Sniḳere, I. Mierina, I. Gehtmane-Hofmane, I. KoroIseva. Overcoming social isolation with digital technologies among ageing populations during Covid-19. SOCIETY. INTEGRATION. EDUCATION. Proceedings of the International Scientific Conference. Volume IV, May 28th-29th 4, 171-178 (2021), DOI:10.17770/sie2021vol4.6356

[12] I. Gehtmane-Hofmane, M. Tēraudkalna, O. Rajevska, I. KoroIseva, I. Reine. Knowledge, attitudes and Covid-19 related behavior among individuals aged 50 and older in Latvia. SOCIETY. INTEGRATION. EDUCATION. Proceedings of the International Scientific Conference 4, 102-112, DOI:10.17770/sie2021vol4.6462 